\title{
DEUTSCHES ORIENT-INSTITUT, HAMBURG
}

In zwangloser Folge erhalten an dieser Stelle Institutionen, die für die Entwicklungspolitik und Entwicklungsforschung von Bedeutung sind, Gelegenheit zur Selbstdarstellung. Diesmal geben wir dem Deutschen-Orient-Institut das Wort.

Das 1960 gegründete DEUTSCHE ORIENT-INSTITUT (DOI) ist das zentrale wissenschaftliche Institut in der Bundesrepublik für den modernen Orient.

Zusammen nit dem DEUTSCHEN INSTITUT FÚR AFRIKAFORSCHUNG, dem INSTITUT FUR ASIENKUNDE, dem INSTITUT FUR IBERO-AMERIKAKUNDE und dem INSTITUT FUR ALLGEMEINE UBERSEEFORSCHUNG ist es in der STIFTUNG DEUTSCHES UBBERSEE-INSTITUT zusammengefaßt, die 1964 auf Anregung des Bundespräsidenten in Hamburg gegründet wurde. Das DOI wird überwiegend vom Senat der Freien und Hansestadt Hamburg und zu einem kleineren Teil vom Bundesministerium für wirtschaftliche Zusammenarbeit in Bonn finanziert. Das Institut befaßt sich in Forschung, Dokumentation und Lehre mit den Regionen Nordafrika und Vorderasien, d. h. allen Mitgliedstaaten der Arabischen Liga, Israel, Türkei, Iran, Afghanistan und Pakistan.

1. Die Forschungstätigkeit ist inter- und multidisziplinär ausgerichtet und hat die gegenwärtigen sozialen, wirtschaftlichen, politischen und kulturellen Entwicklungen in der Region zum Gegenstand. Die Forschungsergebnisse werden, soweit sie nicht für den internen ministerialen Gebrauch bestimmt sind, in der "Schriftenreihe des Deutschen Orient-Instituts" veröffentlicht. Bisher sind 20 Einzelbände veröffentlicht worden (alle im Leske Verlag, Opladen) sowie drei Biographien.

Das DOI ist weiterhin Herausgeber der Vierteljahreszeitschrift ORIENT, die als einzige Zeitschrift in der Bundesrepublik sich ausschließlich mit dem modernen Orient befaßt. Zusammen mit der Forschungsstelle für Völkerrecht und ausländisches öffentliches Recht der Universität Hamburg, mit der Hamburger Gesellschaft für Völkerrecht und auswärtige Politik und mit den übrigen Hamburger Regionalinstituten ist das DOI Mitherausgeber von "Verfassung und Recht in Ubersee“.

Als Mitglied der Stiftung Deutsches Úbersee-Institut ist das DOI auch an der monatlich erscheinenden, englischsprachigen Zeitschrift "Inter Economics“ beteiligt.

Das DOI bezieht etwa 50 Tages- und Wochenzeitungen aus seiner Region in den Originalsprachen, etwa ein Drittel dieser Zeitschriften wird von wissenschaftlichen Referenten ausgewertet und im Archiv des Instituts nach einem detaillierten Schlüssel abgelegt. Die Bibliothek umfaßt rund 10000 gegenwartsbezogene Bände sowie etwa 300 Periodica aus und über die Gesamtregion. An bemerkenswertem Forschungsmaterial sind Gesetzesblätter und Staatsanzeiger aus fast allen Staaten der Region zu nennen, ferner Statistiken, Jahresberichte offizielle und inoffizielle Berichte von staatlichen und nichtstaatlichen Stellen. Im Auftrag der Deutschen Forschungsgemeinschaft sammelt das DOI "Graue Literatur" aus allen Staaten der Region.

2. Dem Institut angeschlossen ist die "DOKUMENTATIONSLEITSTELLE FƯR DEN MODERNEN ORIENT“. Sie erfaßt die Zugänge von 45 Büchereien und 5 bibliographischen Diensten. Ein Stab von Mitarbeitern wertet ca. 300 Zeitschriften aus zu den $Z$ wecken der Literaturdokumentation. Darüber hinaus werden Informa- 
tionen über Entwicklungsprojekte und Institutionen von entwicklungspolitischer Bedeutung gesammelt und aufgeschlossen. Eine gesonderte Kartei erfaßt die nichtstaatlichen Entwicklungshilfeprojekte aller westlichen Länder. Die Dokumentationsleitstelle veröffentlicht alle zwei Monate eine Zugangsliste mit einer Auswahl von jeweils 200 Titeln, stellt die Bibliographie für die Zeitschrift ORIENT zusammen und gibt in unregelmäßigem Abstand Bibliographien zu bestimmten Themen heraus. Auf Anfrage werden Literaturnachweise gegeben.

3. Der Bereich der akademischen Lehre wird durch individuelle Lehraufträge der Universität Hamburg an einzelne Mitarbeiter wahrgenommen.

Das DOI ist der Vorort der „Arbeitsgemeinschaft Vorderer Orient für gegenwärtsbezogene Forschung und Dokumentation", die alle interessierten wissenschaftlichen Institute der Bundesrepublik umfaßt. Archiv, Dokumentations-Leitstelle und Bibliothek des DOI stehen allen Wissenschaftlern zur Verfügung und werden regelmäßig von in- und ausländischen Gastforschern benutzt.

Rainer Büren 